\title{
AVALIAÇÃO DAS ATIVIDADES DA VIDA DIÁRIA DOS PACIENTES COM DOENÇA DE PARKINSON SUBMETIDOS A CIRURGIA ESTEREOTÁXICA
}

\author{
Roberta Arb Saba Rodrigues Pinto', Vanderci Borges², Patrícia Maria C. Aguiar', \\ Fernando A.P. Ferraz ${ }^{4}$, Marcelo Ken-Iti Hisatugo ${ }^{5}$, Henrique Ballalai Ferraz ${ }^{6}$
}

\begin{abstract}
RESUMO - Objetivo: Avaliar o impacto da cirurgia estereotáxica sobre a realização das atividades da vida diária dos paciente com doença de Parkinson (DP). Método: Foram avaliados 30 pacientes com DP forma idiopática submetidos a cirurgia estereotáxica nos períodos pré-operatório, $1^{\circ}, 3^{\circ}, 6^{\circ}$ e $12^{\circ}$ mês pós-operatório, segundo as escala UPDRS - Item II (escala unificada para DP), Schwab \& England e Hoehn \& Yahr. Destes, nove pacientes foram também avaliados no $24^{\circ}$ mês pós-operatório. Resultados: Foram realizadas nove palidotomias pósteroventrais (PPV), sendo duas à esquerda e sete à direita; duas PPV bilaterais no mesmo tempo cirúrgico; 17 talamotomias ventro-laterais (TVL), sendo 12 à esquerda e cinco à direita; duas TVL à esquerda com PPV à direita no mesmo tempo cirúrgico. Os escores médios, na fase off, das escalas utilizadas foram: 65,6 no préoperatório,74 no $1^{\circ}$ mês, 76,6 no $3^{\circ}$ mês, 75,6 no $6^{\circ}$ mês e 72,3 no $12^{\circ}$ mês pós-operatório (Schwab \& England); 21 no pré-operatório, 12,3 no $1^{\circ}$ mês, 14,7 no $3^{\circ}$ mês, 15,27 no $6^{\circ}$ mês e 17,1 no $12^{\circ}$ mês pósoperatório (UPDRS); 3,1 no pré-operatório, 2,8 no $1^{\circ}$ mês, 2,7 no $3^{\circ}$ mês, 2,8 no $6^{\circ}$ mês e 2,85 no $12^{\circ}$ mês pós-operatório (Hoehn \& Yarh). Conclusão: A TVL e a PPV são procedimentos capazes de melhorar a independência dos pacientes para realização das atividades cotidianas, sendo constatado benefício maior nos seis primeiros meses de pós-operatório.
\end{abstract}

PALAVRAS-CHAVE: doença de Parkinson, cirurgia estereotáxica, atividades da vida diária.

\begin{abstract}
Daily living activities in Parkinson's disease patients underwent to stereotactic surgery
ABSTRACT - Objective: To evaluate the impact of stereotactic surgery on daily activities of Parkinson's disease (PD) patients. Method: Thirty patients with idiopathic PD were evaluated before surgery and one, three, six and twelve months after surgery. Patients were evaluated with the UPDRS - part II (Unified Parkinson's Disease Rating Scale) and the Schwab \& England scales. Nine of the patients had also been evaluated after twenty four months. Results: We performed nine posteroventral pallidotomies (PVP), two on the left hemisphere and seven on the right; 17 ventrolateral thalamotomies (VLT), 12 on the left and five on the right; two VLT on the left associated with PVP on the right at the same surgical procedure. The mean "off" phase scores of Schwab \& England scale were: 60.6 before surgery, 74 after the first, 76.6 after the third, 75.6 after the sixth, 72.3 after the twelfth and 71.1 after the twenty fourth months after surgery. The mean "off" phase scores of UPDRS - part II scale were: 21 before surgery, 12.3 after the first, 14.7 after the third, 15.27 after the sixth, 17.1 after the twelfth and 17.5 after the twenty fourth months after surgery. Conclusion: VTL and PVP are useful procedures to improve daily living activities of the PD patients and the best results are seen by six months after surgery.
\end{abstract}

KEY WORDS: Parkinson disease, daily living activities, stereotactic surgery.

A doença de Parkinson (DP) apresenta-se com sintomas como tremor, rigidez e bradicinesia que pioram com a progressão da doença, daí a necessidade de um tratamento eficaz, seja medicamentoso ou cirúrgico. Com o surgimento da cirurgia estereotáxica, na metade do século XX, passou-se a ter uma alternativa terapêutica para o tratamento da DP com menor risco de sequelas. Com o advento da levodopa, tais procedimentos foram colocados em segundo plano, mas, devido à falta de outras substâncias farmacológicas capazes de controlar a DP e às complicações decorrentes do uso prolongado da levo-

Setor de Distúrbios do Movimento, Departamento de Neurologia, Universidade Federal de São Paulo, Escola Paulista de Medicina, São Paulo SP, Brasil (UNIFESP/EPM): ${ }^{1}$ Mestre em Neurologia pela UNIFESP; ${ }^{2}$ Doutora em Neurologia pela UNIFESP; ${ }^{3}$ Mestre em Neurologia pela UNIFESP; ${ }^{4}$ Doutor em Neurocirurgia e Professor Adjunto de Neurocirurgia da UNIFESP; ${ }^{5}$ Mestre em Neurocirurgia pela UNIFESP; ${ }^{6}$ Doutor em Neurologia e Chefe do Setor de Distúrbios do Movimento da UNIFESP.

Recebido 13 Setembro 2001, recebido na forma final 12 Dezembro 2001. Aceito 11 Janeiro 2002. 
dopa, a partir da década de 80, a cirurgia estereotáxica voltou a despertar o interesse nos neurologistas.

De um modo geral, os estudos que avaliam a eficácia da cirurgia na DP, se baseiam nas comparações dos escores motores antes e depois da cirurgia, especialmente segundo a escala UPDRS (Unified Parkinson's Disease Rating Scale - item III). Outras escalas, como a Hoehn \& Yahr e a Schwab \& England, também têm sido utilizadas. Muitos estudos foram realizados avaliando principalmente o aspecto motor, comparando os escores pré e o pós-operatório. Tem sido dada pouca ênfase às atividades da vida diária do parkinsoniano, daí a necessidade de um estudo que analise a evolução dos pacientes submetidos à cirurgia com relação à independência para realizar atividades do dia-a-dia, que está diretamente relacionada com a qualidade de vida dos pacientes parkinsonianos.

O objetivo deste estudo, é avaliar o impacto da cirurgia estereotáxica sobre a realização das atividades da vida diária dos pacientes com DP.

\section{MÉTODO}

Foram avaliados 30 pacientes com diagnóstico de DP, forma idiopática, acompanhados no Ambulatório de Distúrbios do Movimento da Universidade Federal de São Paulo (UNIFESP-EPM), submetidos a tratamento cirúrgico no período de março de 1996 a novembro de 1998. Tal estudo foi aprovado pela Comissão de Ética Médica da UNIFESP. Todos os pacientes submetidos à cirurgia foram informados quanto aos riscos do procedimento, principalmente ao que se refere aos distúrbios da fala e déficits motores.

A - Critérios de indicação cirúrgica (o primeiro é obrigatório, associado à pelo menos um dos outros itens relatados) - 1) diagnóstico de DP idiopática; 2) formas unilaterais ou predominantemente unilaterais, com tremor e rigidez; 3) intolerância às diversas drogas antiparkinsonianas, levando ao comprometimento das atividades da vida diária; 4) presença de flutuações ou discinesias de caráter incapacitantes decorrentes da terapia antiparkinsoniana.

B - Quanto à escolha do alvo cirúrgico eram indicadas - 1) a palidodotomia póstero-ventral (PPV) unilateral quando havia predomínio de bradicinesia ou discinesia no dimídio contralateral e/ou flutuações do efeito da levodopa; 2) PPV bilateral se a bradicinesia e/ou discinesia eram simétricas; 3) a talamotomia ventro-lateral (TVL) unilateral, núcleo ventral intermédio (VIM) e/ou ventral oral posterior (VOP), no lado contralateral ao dimídio mais acometido quando havia predomínio do tremor e da rigidez, nos quadros simétricos, com tremor ou rigidez, optou-se pelo lado que mais beneficiaria o paciente (se destro ou sinistro); 4) TVL de um lado e PPV do outro se quadros mistos (tremor, rigidez, bradicinesia, com ou sem flutuações ou discinesias). As cirurgias bilaterais foram realizadas no mesmo tempo cirúrgico.

C - Escalas utilizadas para avaliação dos pacientes - 1) UPDRS - item II (escore para atividades da vida diária); 2) Hoehn \& Yahr; 3) Schwab \& England. A escala de UPDRS é amplamente aplicada, pois avalia tanto a função motora (parte III), quanto as atividades da vida diária (item II). A escala de Schwab \& England, analisa o grau de dependência para realizar atividades do dia-a-dia dos parkinsonianos em porcentagem e a de Hoehn \& Yahr avalia os pacientes levando em consideração os reflexos posturais, assim como a bilateralidade da doença, especialmente no que se refere à independência para as atividades da vida diária.

D - Momentos de avaliação dos pacientes - Pacientes que utilizavam levodopa foram avaliados no período "on" e "off". O estado de "on" padronizado para o exame era aquele onde o paciente, segundo o seu ponto de vista, apresentava melhor desempenho motor e estava relacionado ao efeito da levodopa ; o oposto ocorria no estado "off". Para avaliação do estado "off", o paciente deveria estar sem o uso de levodopa por, no mínimo, 12 horas. Períodos de avaliação: pré-operatório, $1^{\circ}, 3^{\circ}, 6^{\circ}, 12^{\circ} \mathrm{e}$ $24^{\circ}$ mês de pós-operatório.

E - Procedimento cirúrgico - O aparelho utilizado para a estereotáxia foi o ETMB03 (Micromar Ind. \& Com. Ltda). No dia do procedimento, foi fixado o aro estereotáxico em três pontos, um frontal mediano e dois occipitais. Depois deste procedimento, os pacientes foram submetidos à tomografia computadorizada de crânio (TC), para o cálculo das coordenadas estereotáxicas. Após a determinação das coordenadas estereotáxicas os pacientes foram conduzidos ao centro cirúrgico e, após anestesia local, trepanação manual e abertura da dura mater, foi introduzido até o alvo, um eletrodo ligado por meio de um termistor ao aparelho de rádiofrequência, o qual permitia a produção de lesões com duração e temperatura determinadas. As lesões talâmicas foram realizadas com os seguintes parâmetros físicos: $70^{\circ}$, durante 60 segundos, tanto no VIM quanto no VOP. Os mesmos parâmetros foram utilizados para a lesão do Gpi. Caso fosse detectado algum distúrbio da fala ou paresias, o procedimento era interrompido.

F - Método estatístico - para análise dos resultados foram aplicados os seguintes testes - 1) análise de variância por postos de Friedman ${ }^{1}$ com a finalidade de comparar as escalas nos períodos pré-operatório, $1^{\circ}, 3^{\circ}, 6^{\circ}, 12^{\circ}$ mês de evolução; esta análise foi complementada pelo teste de comparações múltiplas; 2) teste de Wilconxon'1, para comparar as fases "on" e "off" dos pacientes em cada um dos períodos estudados; Fixou-se em 0,05 ou $5 \%(\alpha \leq$ $0,05)$ o nível de rejeição da hipótese de nulidade assinalando-se com um asterisco os valores significantes. 
Tabela 1. Perfil da amostra dos 30 pacientes submetidos à cirurgia estereotáxica e procedimentos realizados.

\begin{tabular}{ll}
\hline idade média & 55,2 anos (42-68 anos) \\
sexo & 17 masc / 13 fem \\
procedimento realizado & 9 PPV unilateral (2 esq / 7 dir) \\
& 2 PPV bilateral \\
& 17 TVL (12 esq / 5 dir) \\
& 2 TVL esq + PPV dir \\
\hline
\end{tabular}

\section{RESULTADOS}

O perfil dos pacientes estudados consta da Tabela 1.

\section{Complicações}

Entre os pacientes estudados, 7 apresentaram complicações, e destes apenas dois não tiveram meIhora completa.

Dentre os 19 pacientes submetidos a talamotomia, um apresentou paralisia facial central e disartria, outro apresentou hemiparesia e dois apresentaram desorientação têmporo espacial e confusão mental.

Dos 13 pacientes submetidos a palidotomia, um mesmo paciente apresentou paralisia facial periférica, disartria, alucinação, urgência miccional e hipofonia. Outros dois pacientes, apresentaram hipofonia e um deles tinha também, quadrantanopsia superior.

\section{Escore médio}

Os escores médos obtidos constam de Tabela 2 e Figura 1.

\section{Dose da levodopa}

A análise da variação da dose de levodopa do período pré-operatório, $12^{\circ}$ e $24^{\circ}$ mês pós-operatório mostra que não houve modificação, isto provavelmente está relacionado ao perfil clínico de cada paciente. Em uma parte dos casos é possível promover diminuição da dose, após o procedimento cirúrgico, naqueles pacientes que passem a apresentar períodos "on" mais prolongados, isto é, diminuição das flutuações. Por outro lado, para aqueles que apresentam discinesias incapacitantes no período pré-operatório, com a cirurgia, foi possível aumentar a dose de levodopa, obtendo períodos "on" mais prolongados e com menos discinesias.

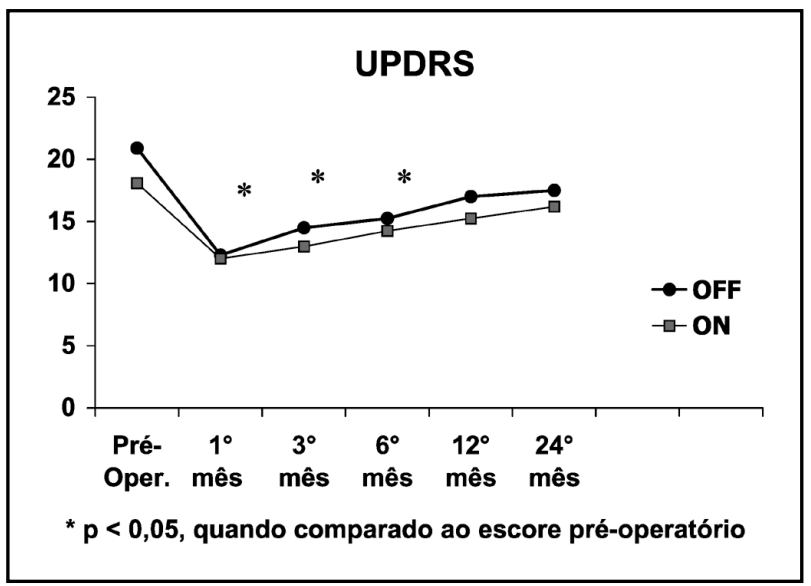

Fig 1. Escores médios da escala UPDRS no $1^{\circ}, 3^{\circ}, 6^{\circ}, 12^{\circ}$ e $24^{\circ}$ mês pós-operatório.

Tabela 2. Escores médios dos pacientes, utilizando as escalas Schwab \& England, UPDRS no período on e off e Hoehn \& Yahr no período on e off.

\begin{tabular}{|c|c|c|c|c|c|c|}
\hline Escores médios & Pré-op & $1^{\circ}$ mês & $3^{\circ}$ mês & $6^{\circ}$ mês & $12^{\circ}$ mês & $24^{\circ}$ mês \\
\hline Schwab \&England & 66,1 & 74 & 71,1 & 75,7 & 73,6 & 71,1 \\
\hline UPDRS período on & 18,1 & 12 & 13 & 14,25 & 15,25 & 16,2 \\
\hline UPDRS período off & 20,9 & 12,3 & 14,5 & 15,25 & 17 & 17,5 \\
\hline Hoehn \& Yahr período on & 3,03 & 2,78 & 2,71 & 2,78 & 2,78 & 3,3 \\
\hline Hoehn \& Yahr período off & 3,1 & 2,78 & 2,73 & 2,8 & 2,58 & 3,3 \\
\hline \multicolumn{7}{|l|}{ Análise estatística } \\
\hline \multicolumn{7}{|c|}{ Schwab \& England - escores médios pré-op $<3^{\circ}$ e $6^{\circ}$ mês pós-op $(p<0,05)$} \\
\hline \multicolumn{7}{|c|}{ UPDRS ON - pré-op > $1^{\circ}, 3^{\circ}$ e $6^{\circ}$ mês pós-op $(p<0,05)$} \\
\hline \multicolumn{7}{|c|}{ UPDRS OFF - pré-op $>1^{\circ}, 3^{\circ}$ e $6^{\circ}$ mês pós-op $(p<0,05)$} \\
\hline \multicolumn{7}{|c|}{ UPDRS on X off - não foi estatisticamente significante } \\
\hline \multicolumn{7}{|c|}{ Hoehn \& Yahr on - pré-op $>1^{\circ}, 3^{\circ}, 6^{\circ}$ e $12^{\circ}$ mês pós-op $(p<0,05)$} \\
\hline \multicolumn{7}{|c|}{ Hoehn \& Yahr off - pré-op $>1^{\circ}, 3^{\circ}$ e $6^{\circ}$ mês pós-op $(p<0,05)$} \\
\hline
\end{tabular}




\section{Doses médias de levodopa}

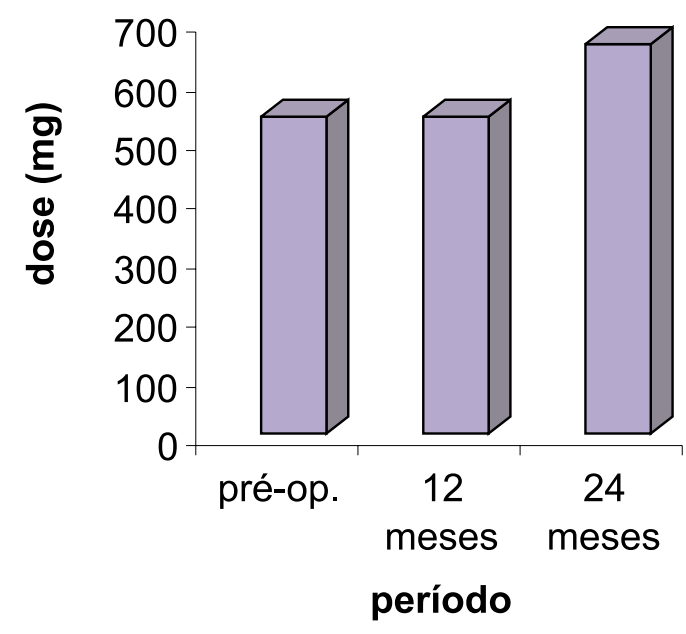

Fig 2. Doses médias de levodopa. Não houve diminuição da dose média de levodopa estatisticamente significante.

\section{DISCUSSÃO}

Foram estudados 30 pacientes com DP, forma idiopática, submetidos a tratamento cirúrgico devido a má resposta ao tratamento medicamentoso, seja por intolerância aos medicamentos antiparkinsonianos ou pela presença de flutuações e discinesias causadas por estas drogas. Todos estes fatores levam ao comprometimento da qualidade de vida dos pacientes.

Em sua maioria, os protocolos que analisam a meIhora dos pacientes parkinsonianos submetidos a tratamento cirúrgico utilizam a escala UPDRS, levando muito em consideração o desempenho motor (item - III) mostrando melhora da função motora ${ }^{2-6}$ Entretanto, poucos são os estudos que dão ênfase às atividades da vida diária, aspecto de grande importância para os parkinsoniamos, uma vez que, estão relacionadas à independência destes indivíduos. Uma forma de avaliar este aspecto, é com a escala UPDRS (item - II) para atividades da vida diária, a escala de Schwab \& England e a escala de Hoehn \& Yahr.

Os pacientes da nossa amostra que utilizavam levodopa (28 pacientes) foram avaliados no estado de "on" e "off". Os pacientes foram seguidos com maior frequência nos primeiros 6 meses de pós-operatório, período este em que, segundo experiência obtida com outros pacientes já operados no nosso serviço, se observam maiores modificações. No sétimo dia de pós-operatório ocorreu o primeiro atendimento para verificar se era necessário ajuste da dose dos medicamentos utilizados. Posteriormente, os pacientes foram avaliados no $1^{\circ}, 3^{\circ}, 6^{\circ}$ e $12^{\circ}$ meses após a cirurgia; 9 dos 30 pacientes foram, também, reavaliados no $24^{\circ}$ mês pós-operatório.

Vários estudos são a favor da cirurgia ablativa na DP; porém, a sua indicação deve ser feita àqueles pacientes com comprometimento para realização das atividades diárias e que estejam, de alguma forma, dependentes ${ }^{7-13}$. A escolha do alvo cirúrgico neste estudo segue a tendência atual da literatura, que indica a PPV nos casos nos quais a bradicinesia, flutuações decorrentes do uso da levodopa e ocorrência de discinesias são predominantes ${ }^{2-4,12,14-19}$. A TVL é indicada nos casos onde há predomínio da rigidez e do tremor ${ }^{20-24}$. Nosso estudo assemelha-se muito com o de Ferraz et al. ${ }^{25}$, quanto à técnica cirúrgica e escolha do alvo cirúrgico.

$\mathrm{Na}$ análise da variação da dose de levodopa por paciente com DP no período pré-operatório, $12^{\circ} \mathrm{e}$ $24^{\circ}$ mês, após o procedimento cirúrgico, observamos que não houve modificação estatísticamente significante na dose de levodopa utilizada pelos pacientes antes e depois da cirurgia (Fig 2). A explicação para esta estabilidade da dose se deve a vários fatores. A variação da dose de levodopa após a cirurgia está relacionada ao perfil clínico de cada paciente. Em uma parte dos casos, é possível promover uma diminuição da dose, após o procedimento cirúrgico nos pacientes que passem a apresentar períodos "on" mais prolongados, isto é, diminuição das flutuações. Por outro lado, para aqueles pacientes que apresentavam discinesias incapacitantes no período pré-operatório, com a cirurgia é possível aumentar a dose de levodopa, obtendo-se períodos "on" mais prolongados e com menos discinesias.

Estes resultados assemelham-se aos de outros estudos realizados, em que também não houve mudança estatisticamente significante na dose de levodopa $a^{3,4,6,16,26-28}$.

$\mathrm{Na}$ análise realizada por Masterman et al., que avaliaram pacientes parkinsonianos submetidos a palidotomia unilateral no $3^{\circ}$ e $6^{\circ}$ mês pós-operatório, $44 \%$ dos pacientes mantiveram a mesma dose de levodopa, $41 \%$ tiveram a dose diminuída e $15 \%$ necessitaram de aumento na dose de levodopa ${ }^{5}$. Estudo realizado por Dogali et al. ${ }^{3}$ e outro por Laitinen ${ }^{14}$ mostraram diminuição na dose da levodopa no período pós-operatório. Esses dados não foram confirmados pela maioria dos estudos, incluindo o nosso.

A análise dos escores médios segundo a escala de Schwab \& England (Tabela 2) mostra melhora no 
grau de independência dos pacientes submetidos à cirurgia, especialmente no $3^{\circ}$ e $6^{\circ}$ mês pós-operatório em relação ao período pré-operatório. Em porcentagem, houve melhora no grau de independência destes pacientes de $66,1 \%$ no pré-operatório para $77,1 \%$ no $3^{\circ}$ mês e $75,7 \%$ no $6^{\circ}$ mês.

$\mathrm{O}$ fato de não haver melhora significante no $1^{\circ}$ mês pós-operatório, provavelmente se deve ao período de adaptação e recuperação do paciente do procedimento cirúrgico. Além disso, a escala de Schwab \& England avalia as atividades da vida diária de forma subjetiva, isto é, o quanto o paciente se considera capaz e independente, diferente da avaliação feita para função motora, que é objetiva (exame físico). Estudo realizado por Baron et al., também mostra melhora quanto à independência dos pacientes submetidos a palidotomia no $3^{\circ}, 6^{\circ}$ e $12^{\circ}$ mês pósoperatório ${ }^{26}$. O benefício cirúrgico também foi cons-

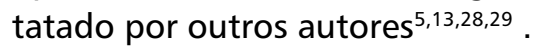

Em relação ao período pré-operatório, a análise dos escores médios das escalas de Horhn \& Yahr, mostra benefício cirúrgico no $1^{\circ}, 3^{\circ}, 6^{\circ}$ e $12^{\circ}$ mês pós-operatório no período "on" e melhora no $1^{\circ}, 3^{\circ}$ e $6^{\circ}$ mês pós-operatório no período "off".

Masterman publicou estudo com pacientes submetidos a PPV unilateral que foram avaliados no $3^{\circ}$ e $6^{\circ}$ mês pós-operatório, também relatando melhora estatisticamente significante, pela escala de Hoehn \& Yahr tanto em "on" quanto em "off" . A análise da escala de Hoehn \& Yahr de 126 pacientes, realizada por lacono durante o primeiro ano pós-operatório, mostra diminuição do escore na fase "on", evidenciando melhora destes pacientes ${ }^{2}$. Já Shannon, analisando pacientes submetidos a palidotomia, encontrou benefício cirúrgico transitório no primeiro mês pós-operatório na fase "off", que não se manteve no $6^{\circ}$ mês $^{30}$.

Ressaltamos, mais uma vez que a escala modificada de Hoehn \& Yahr, utilizada para graduar a atividade da DP, não reflete muito bem o grau de meIhora dos pacientes submetidos à cirurgia. Isto se deve ao fato de poder haver melhora do tremor e da rigidez e, consequentemente, maior capacidade para realizar as atividades cotidianas, mas o paciente pode ainda manter a bradicinesia e alteração dos reflexos posturais, mantendo o mesmo grau nesta escala. Portanto, ela não deve ser utilizada com o objetivo de avaliar a capacidade para o desempenho nas atividades da vida diária e, além disso, modificações muitas vezes significantes não reproduzem mudanças nos escores.
A Tabela 2 evidencia a melhora dos escores da escala UPDRS (item II - atividades da vida diária), no $1^{\circ}, 3^{\circ}, 6^{\circ}$ e $12^{\circ}$ mês pós-operatório, tanto na fase "on" quanto na fase "off". Ela mostra diminuição do escore total dos pacientes no $1^{\circ}, 3^{\circ}, 6^{\circ}$ mês pósoperatório, evidenciando uma boa resposta ao tratamento cirúrgico. O benefício da cirurgia no $12^{\circ} \mathrm{e}$ $24^{\circ}$ pós-operatório não é significante do ponto de vista estatístico. Observamos que, após o $6^{\circ}$ mês pósoperatório, há tendência à manutenção nos valores do escore total, em relação ao período pré-operatório, talvez pelo fato de progredir a doença em relação aos meses anteriores. Após o $6^{\circ}$ mês a escala detecta perda do benefício inicial da cirurgia igualando os escores do pré-operatório, até o $24^{\circ}$ mês. Não temos como saber se os valores iguais dos escores a partir do $6^{\circ}$ mês representam mesmo uma perda do benefício ou se a cirurgia contribuiria, pelo menos, para evitar a progressão mais rápida da doença.

Estudo realizado por Dogali et al. ${ }^{3}$, compara um grupo de pacientes submetidos a palidotomia a outro, submetido a tratamento medicamentoso. Até o $1^{\circ}$ ano de seguimento, observou-se melhora significante quanto à independência para as atividades da vida diária dos pacientes; após este período, observa-se tendência de manutenção do benefício cirúrgico, sugerindo que a palidotomia poderia retardar a progressão da DP, pois, quando se compara com o grupo não operado, observa-se maior comprometimento e progressão da doença .Uma explicação para o suposto efeito neuroprotetor da cirurgia sobre a DP seria que a palidotomia ou a talamotomia acabaram levando a uma diminuição da atividade exitatória sobre a substância negra, o que resultaram em diminuição da excitoxicidade.

Lang et al. ${ }^{27}$ analisaram 40 pacientes submetidos a palidotomia, observando benefício cirúrgico, tanto para função motora, quanto para as atividades diárias (escala UPDRS - item II). A maioria deles $(n=39)$ foi avaliada no $6^{\circ}$ mês pós-operatório e no $1^{\circ}$ ano de cirurgia $(n=27)$, sendo uma menor parte acompanhados até o $2^{\circ}$ ano pós-operatório. No $6^{\circ}$ mês pós-operatório, na fase "off", $30 \%$ dos pacientes tornaram-se mais independentes para realizarem as atividades diárias o que persistiu até o $2^{\circ}$ ano de cirurgia. Na fase "on", este benefício ocorreu em 20\% dos pacientes, possivelmente pela redução das discinesias ${ }^{27}$. Outros estudos também corroboram com estes resultados $6,16,31-33$.

lacono et al. ${ }^{2}$, por sua vez, notaram que a melhora se manteve na fase "on" em $70 \%$ dos 126 pacien- 
tes analisados. O estudo realizado por Baron et al. ${ }^{26}$ mostrou que, na fase "off", houve diminuição do escore para as atividades da vida diária, com melhora de $34 \%$ dos pacientes operados, no $3^{\circ}, 6^{\circ}$ e $12^{\circ}$ mês pós-operatório. Na fase "on", a melhora foi de $28,5 \%$ somente no $3^{\circ}$ mês pós-operatório.

Uitti et al. ${ }^{4}$ e Scott et al. ${ }^{29}$, analisaram num curto prazo de 3 meses a variação do escore da escala UPDRS - item II de pacientes que realizaram palidotomia, constatando melhora estatisticamente significante no $3^{\circ}$ mês após o procedimento, tanto na fase "on" como na "off". Esta melhora no desempenho nas atividades da vida diária não foi observada no estudo realizado por Melnick et al. ${ }^{19}$, que acompanharam 29 pacientes submetidos a palidotomia , durante 6 meses. Este estudo mostrou apenas meIhora na função motora. Não temos uma explicação para a discordância deste estudo em relação ao nosso e aos demais. Melnick et al. ${ }^{19}$ tentam explicar a partir do fato de que a avaliação das atividades da vida diária é feita pelo próprio paciente de maneira subjetiva, enquanto que o exame da função motora, é feito pelo examinador de modo objetivo.

Entre os nossos pacientes estudados, sete apresentaram complicações e destes, apenas dois não tiveram melhora completa. Devido ao pequeno número de procedimentos bilaterais em relação aos unilaterais, não foi possível concluir se o número de complicações é maior nos procedimentos bilaterais do que nos unilaterais.

As complicações que ocorreram neste estudo estão de acordo com dados encontrados na literatura. Perry e Lenz ${ }^{24}$ apresentam uma revisão de trabalhos realizados com pacientes submetidos a TVL que apresentavam complicações transitórias, dentre elas: disartria, disfasia, hemiparesia e alterações mentais. Outro trabalho também apresenta resultado semeIhante ${ }^{21}$.

Quanto a PPV, os estudos mostram que as complicações mais encontradas são: disartria, confusão mental, alterações cognitivas, alteração de campo visual, disfagia e hemiparesia ${ }^{2,4,12,14-16,32}$. De acordo com o objetivo deste estudo, avaliou-se o benefício que a cirurgia estereotáxica proporciona aos pacientes parkinsonianos, quando se analisa o grau de independência para realizar tarefas do dia-a-dia. Usando a mesma metodologia empregada neste estudo, nosso grupo também observou melhora no desempenho motor medido pelo UPDRS- item $\mathrm{III}^{6}$. A cirurgia contribui para melhora das flutuações, com diminuição dos períodos na fase "off", levando a me-
Ihora na execução das atividades diárias e, portanto, benefício na qualidade de vida destes pacientes.

Nos últimos anos, a qualidade de vida dos pacientes com DP tem sido considerada de grande importância, e novos estudos e escalas surgiram para melhor análise $\mathrm{e}^{24,34-36}$. As escalas mais frequentemente utilizadas são a PDQ - 39 (Parkinson's Disease Questionaire) e a SF - 36 $6^{37}$. Tais escalas levam em consideração, entre outros itens, a mobilidade, as atividades da vida diária e o bem estar social e emocional dos pacientes analisados.

Estudo recente, realizado por Martínez-Martín et al. ${ }^{28}$, analisa a qualidade de vida de pacientes submetidos a palidotomia, no $3^{\circ}$ mês pós-operatório, constatando melhora da função motora, especialmente na fase "off", assim como, das flutuações das atividades da vida diária e consequentemente da qualidade de vida destes pacientes, uma vez que existe íntima relação entre estes fatores.

Em conclusão, nosso estudo mostra que a cirurgia estereotáxica para doença de Parkinson é um procedimento capaz de melhorar a independência dos pacientes nas tarefas cotidianas, especialmente nos primeiros 6 meses após o procedimento, sem estar associado a complicações graves e duradouras. Apesar do nosso estudo demonstrar clara meIhora nas atividades da vida diária, novos estudos devem ser realizados, dando maior ênfase à qualidade de vida dos parkinsonianos.

\section{REFERÊNCIAS}

1. Siegel S, Castellan NJ Jr. Nonparametric statistics second edition. New York: McGraw-Hill int.ed 1988:399.

2. Iacono RP, Shima F, Lonser R, Kiniyosh S, Maeda G, Yamada S. The results, indications, and phisiology of posteroventral pallidotomy for patients with Parkinson's disease. Neurosurgery 1995; 36 : 1118 - 1127.

3. Dogali M, Fazzini E, Kolodnye, et al. Stereotatic ventral pallidotomy for Parkinson's disease: a long- term follow- up of unilateral pallidotomy. Neurology 1995; 45:753-761.

4. Uitti RJ, Wharen RE, Turk MSN, et al. Unilateral pallidotomy for Parkinson's disease: comparison of outcome in younger versus elderly patients. Neurology 1997; 49:1072-1077.

5. Masterman D, Salles A, Baloh RW, et al. Motor cognitive and behavioral performance following unilateral ventro-posterior pallidotomy for Parkinson's disease. Ann Neurol 1998; 55:1201-1208.

6. Aguiar PMC. Talamotomia centro-lateral e palidotomia póstero-ventral no tratamento de doença de Parkinson : avaliação do desempenho motor no decorrer de doze meses. Tese de Mestrado, UNIFESP. São Paulo,1999.

7. Kelly PJ, Gillignhan FJ. The long term results of stereotxic surgery and L-dopa therapy in patients with Parkinson's disease. J Neurosurg 1980; 53:332-337.

8. Niclot $\mathrm{P}, \mathrm{N}^{\prime}$ guyen JP, Cesaro $\mathrm{P}$, Degos JD. Traitment du tremblement par chirurgie stéréotaxique. Rev Neurol 1993; 149:755-763.

9. Vitek J, Bakay RAE, Delong MR. Microelectrode-guided pallidotomy for medically intractable Parkinson's disease. Adv Neurol 1997;74:183-198.

10. Jankovic J, Hamilton WJ, Grossman RG. Thalamic surgery for movement disordesr. Adv Neurol 1997;74:221-233.

11. Lai EC, Krauss JK. Indications for pallidal surgery for Parkinson's disease. In: Parkinson's disease and movement disorders. Philadelphia: Lippincott-Raven Publishers, 1998:113-120. 
12. Lang AE, Lozano AM. Parkinson's disease. N Engl J Med 1998;339:11301143.

13. Obeso JA, Guriti J, Alvarez L, Macias R, Linasoro G. Ablative surgery for Parkinson's disease. In Jankovic J, Tolosa E, (eds). Parkinson's disease and movement disorders. 3.Ed. Media: Williams \& Wilkins, 1998:1049-1064.

14. Laitinen LV, Bergheim AT, Hariz MI. Leksell's posteroventral pallidotomy in the treatment of Parkinson's disease. J Neurosurg 1992;76:53-61.

15. Laitinen LV. Pallidotomy for parkinson's disease. Neurosurg Clin N Am 1995;6:15-112.

16. Fazzini E, Dogali M, Stereo D, Eidelberg D, Beric A. Stereotatic pallidotomy for Parkinson's disease - a long-term follow- up of unilateral pallidotomy. Neurology 1997;48:1273 - 1277.

17. Johanson F, Malm J, Nordh E, Hariz M. Usefulness of pallidotomy in advanced Parkinson's disease. J Neurol Neurosurg Psichiatry 1997; 62:125 - 132.

18. Kishore A, Turnbull IM, Snow BJ, et al. Efficacy, stability and predictors of outcome of pallidotomy for Parkinson's disease: six - month followup with aditional 1 - year observations. Brain 1997; 120:729-737.

19. Melnick ME, Dowling GA, Aminoff MJ, Barbaro NM. Effect of pallidotomy on postural control and motor function in Parkinson disease. Arch Neurol 1999; 56:1361-1365.

20. Kelly PJ, Ahlskog JE, Goerss SJ, Daube JR, Duffy JR, Kall BA . Computer - assisted stereotactic ventralis lateralis thalamotomy with microelectrode recording control in patients with Parkinson's disease. Mayo Clin Proc 1987;62:655-664.

21. Nagaseki $Y$, Shibazaki T, Hirai T, Kawashima Y, Hirato M, Wada H. Long-term follow-up results of VIM - thalamotomy. J Neurosurg 1986;65:296-302.

22. Fox MW, Ahloskog JE, Kelly PJ. Stereotatic ventrolaterais thalamotomy for medically refractory tremor in post - levodopa era Parkinson's disease patients. J Neurosurg 1991;75:723-730.

23. Diederich N, Goetz CG, Stebbins GT, et al . Blinded evaluation confirms long-term assymetric effect of unilateral thalamotomy or subthalamotomy or tremor in Parkinson's disease. Neurology 1992;42:1311-1314.

24. Perry VL, Lenz FA. Ablative therapy for movement disorders: thalamotomy for Parkinson's disease. Neurosurg Clin N Am 1998;9:317-323.
25. Ferraz FP, Kent-Iti M, Ferraz HB, Aguiar PMC, Bidó JOF. Talamotomia e palidotomia estereotáxica com planejamento computatorizado no tratamento da doença de Parkinson. Arq Neuropsiquiatr 1998;56:789-797.

26. Baron MS, Vitek JL, Bakay RAE, Green J, Kaneoke Y, Hashimoto T. Treatment of advanced Parkinson's disease by posterior GPi pallidotomy: 1-year results of a pilot study. Ann Neurol 1996; 40:355-366.

27. Lang AE, Lozano AM, Duff J, et al. Medial pallidotomy in late stage of Parkinson's disease and striatalnigral degeneration. Adv Neurol 1997; 74:199-211.

28. Martinez-Martin P, Valdeoriola F, Molinuevo JL, Nobbe FA, Rumia J, Tolosa E . Pallidotomy and quality of life in patients with Parkinson's disease: an early study. Mov Disord 2000;15:65-70.

29. Scott R, Gregory R, Hines N, et al. Neuropsychological, neurological and functional outome following pallidotomy for Parkinson's disease. Brain 1998;121:659 -675

30. Shannon KM, Penn RD, Kroin JS, et al. Stereotactic pallidotomy for the treatment of Parkinson's disease: efficacy and adverse effects at 6 months in 26 patients. Neurology 1998;50:434-438.

31. Starr PA, Vitek JL, DeLong M, Mewes K, Bakay RAE. Pallidotomy: theory and technique.Tech Neurosurg 1999;5:31-45

32. Lozano AM, Lang AE, Gaivez-Jimenez N. Assessing the effects of Pallidotomy in parkinson's disease. Lancet 1995;347:25.

33. Kumar K, Lozano AM, Montgomery E, Lang AE. Pallidotomy and deep brain stimulation of the Pallidum and subtalamic nucleus in advanced Parkinson's disease. Mov Disord 1997;13(Suppl 1):73-82.

34. Fukunaga $\mathrm{H}, \mathrm{Kasai} \mathrm{T}$, Yoshidome $\mathrm{H}$. Clinical firdings, status of care, comprehensive quality of life, daily life therapy and treatment at home in patients with Parkinson's disease. Eur Neurol 1997;38(Suppl 2): 64-69.

35. Tandberg E, Larsen JP, Karlsen K.A community based study of sleep disorders in patients with parkinson's disease. Mov Disord 1998;13:895-899.

36. Karlsen KH, Larsen JP, Tandberg E, Maeland JG. Influence of clinica and demographic variables on quality of life in Patients with parkinson's disease. J Neurol Neurosurg Psychiatry 1999;66:431-435.

37. Fitzpatrick R, Peto V, Tenkinson C, Greenhall R, Hyman N. Healthrelated quality of life in Parkinson's disease: a study of out patient clinic attenders. Mov Disord 1997;12:916-922. 